\title{
DOUBLY PERIODIC SETS OF THIN BRANCHED INCLUSIONS IN THE ELASTIC MEDIUM: STRESS CONCENTRATION AND EFFECTIVE PROPERTIES
}

\author{
Heorhiy SULYM*, Iaroslav PASTERNAK**, Serhiy KUTSYK**, Wojciech GRODZKI* \\ * Bialystok Technical University, Wiejska Str. 45C, 15-351 Bialystok, Poland \\ ** Lutsk National Technical University, Lvivska Str. 75, 43018 Lutsk, Ukraine \\ h.sulym@pb.edu.pl, pasternak@ukrpost.ua, sergosidpr@bigmir.net, wojtekgrr@interia.pl
}

\begin{abstract}
This paper considers the doubly periodic problem of elasticity for anisotropic solids containing regular sets of thin branched inclusions. A coupling principle for continua of different dimension is utilized for modeling of thin inhomogeneities and the boundary element technique is adopted for numerical solution of the problem. The branches of the inclusion can interact both inside the representative volume element and at the interface of neighbor representative elements. A particular example of the elastic medium reinforced by a doubly periodic set of I-beams is considered. Stress intensity and stress concentration inside and outside thin inclusions are determined. The dependence of the effective mechanical properties of the reinforced composite material on the volume fraction of the filament and its rigidity is obtained.
\end{abstract}

Key words: Anisotropic, Doubly Periodic, Thin Branched Inclusion, Reinforcement, Effective Modulus

\section{INTRODUCTION}

One of the important tasks of mechanics of inhomogeneous solids, in particular the theory of thin inclusions (Sulym, 2007), is the study of stress concentration at the reinforcements in the concrete and composite materials. The corresponding reinforcements are commonly produced using the standard shapes (a T-beam, an I-beam, a channel, and angle etc.), which have the shape of non-smooth thin branched inclusions. The solution of elasticity problems for solids containing doubly or triply periodic sets of thin inhomogeneities also allows estimating the effective mechanical properties of the corresponding composite materials. Moreover, it is possible to consider both cases of interaction of reinforcements: inside a representative volume element, and on its boundary. The latter allows modeling of novel continuously reinforced composite materials, which strength and rigidity should be very high due to their solidity and the absence of high stress concentration.

The analysis of elastic crooked or cross-like inclusions was mainly performed for the aperiodic problems of elasticity. In particular, the papers (Osiv and Sulym, 2001; 2002) considered the antiplane deformation of solids containing a set of arbitrarily oriented rectilinear joined ribbon-like elastic inclusions. Šackyj and Kundrat (2004) provided the analysis of the out-of-plane deformation of an isotropic medium containing a set of joined rigid line inclusions, in particular, those with the shape of and angle, a T-beam, an I-beam etc. The plane problem of elasticity was considered for an infinite medium containing a cross-like rigid line inclusion (Antipov et al., 1987; Popov, 1993) and a flexible crosslike elastic inclusion (Grigoryan et al., 2002). Pasternak and Sulym (2011) were the fist to propose the general boundary element approach for the solution of plane problems of elasticity for isotropic and anisotropic solids containing systems of joined multibranched thin elastic inclusions.

The study of doubly periodic sets of thin inhomogeneities was held in Refs. (Kosmodamianskij, 1976; Dolgikh and Fil'shtinskii, 1979; Pasternak, 2012; Pasternak and Sulym 2013). However, to the best of authors' knowledge the doubly periodic problems for thin branched inclusions were not referenced in the scientific literature. Therefore, this paper considers the general approach for studying the doubly periodic problems for anisotropic elastic solids containing thin branched inclusions. This approach is based on the coupling principle for continua of different dimension and the boundary element method.

\section{PROBLEM FORMULATION BASED ON THE COUPLING PRINCIPLE}

Consider an infinite anisotropic elastic medium containing a doubly periodic set of thin elastic branched inclusions identical to each other, such that translation symmetry conditions are satisfied both for geometrical and physical properties of the solid. For modeling of the corresponding composite material the coupling principle for continua of different dimension (Sulym, 2007) is used. Thus, the inclusion is replaced with the line $\Gamma_{C}$ of field discontinuities. One can assume that the line $\Gamma_{C}$ coincides with a median line of the thin inhomogeneity. Thus, using this technique the problem is split into two sub-problems: an external one, which considers the stress-strain state of a solid and its effective mechanical properties due to a set of discontinuity lines, and an internal one, which is focused on the modeling of relations between displacements and tractions at the opposite sides of the thin inhomogeneity, and thus, at the opposite faces of the discontinuity line $\Gamma_{C}$.

According to Pasternak (2012), the external problem is reduced to the following system of dual integral equations: 


$$
\begin{aligned}
& \frac{1}{2} \Sigma \mathbf{u}^{0}(\mathbf{y})=\operatorname{RPV} \int_{\Gamma_{0}^{+}} \mathbf{U}^{\mathrm{dp}}(\mathbf{x}, \mathbf{y}) \Sigma \mathbf{t}^{0}(\mathbf{x}) d \Gamma(\mathbf{x}) \\
&-\mathrm{CPV} \int_{\Gamma_{0}^{+}} \mathbf{T}^{\mathrm{dp}}(\mathbf{x}, \mathbf{y}) \Delta \mathbf{u}^{0}(\mathbf{x}) d \Gamma(\mathbf{x})+\mathbf{I}^{\infty}(\mathbf{y}), \\
& \frac{1}{2} \Delta \mathbf{t}^{0}(\mathbf{y})=n_{j}^{+}(\mathbf{y})\left[\mathrm{CPV} \int_{\Gamma_{0}^{+}} \mathbf{D}_{j}^{\mathrm{dp}}(\mathbf{x}, \mathbf{y}) \Sigma \mathbf{t}^{0}(\mathbf{x}) d \Gamma(\mathbf{x})\right. \\
&\left.-\operatorname{HPV}_{\Gamma_{0}^{+}} \mathbf{S}_{j}^{\mathrm{dp}}(\mathbf{x}, \mathbf{y}) \Delta \mathbf{u}^{0}(\mathbf{x}) d \Gamma(\mathbf{x})+\mathbf{\Xi}_{j}^{\infty}\right],
\end{aligned}
$$

where $\mathbf{u}^{0}$ and $\mathbf{t}^{0}$ are displacement and traction vectors at the base contour $\Gamma_{0} \cup \Gamma_{C}$ in the selected representative volume element; $\Delta(\cdot)=(\cdot)^{+}-(\cdot)^{-}, \sum(\cdot)=(\cdot)^{+}+(\cdot)^{-}$; signs "+" and "" denote variables concerned with faces $\Gamma_{C}^{+}$and $\Gamma_{C}^{-}$of the mathematical cut $\Gamma_{C}$; symbols RPV stands for the Riemann Principal Value, CPV for the Cauchy Principal Value and HPV for the Hadamard Principal Value (finite part) of an integral. The functions $\mathbf{I}^{\infty}(\mathbf{y})$ and $\mathbf{\Xi}_{j}^{\infty}(\mathbf{y})$ define the external load set by the components $\left\langle\sigma_{i j}\right\rangle$ of the average stress tensor and are given by special integral identities (Pasternak, 2012). Closed-form expressions for the kernels $\mathbf{K}^{\mathrm{dp}}=\left[\mathbf{U}^{\mathrm{dp}}, \mathbf{T}^{\mathrm{dp}}, \mathbf{D}_{j}^{\mathrm{dp}}, \mathbf{S}_{j}^{\mathrm{dp}}\right]$ are given in Ref. (Pasternak, 2012). These kernels are functions of coordinates of collocation point $\mathbf{y}$ and integration point $\mathbf{x}$, and also depend on the vectors $\boldsymbol{\omega}^{(1)}$ and $\boldsymbol{\omega}^{(2)}$ of the fundamental periods, which form the lattice (Fig. 1).

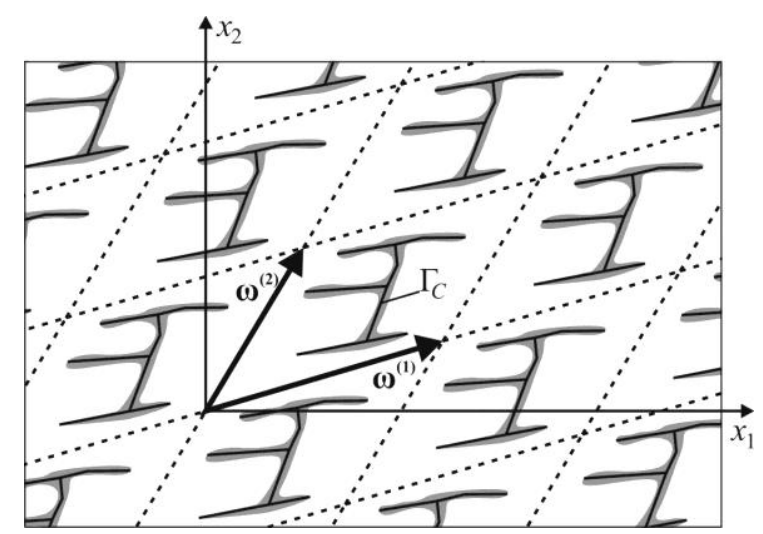

Fig. 1. The sketch of the problem

Pasternak and Sulym (2011), Pasternak (2011) provide the closed-form relations for the mathematical models of thin nonbranched elastic and piezoelectric, isotropic and anisotropic inclusions, which can be generally presented with the following functional dependences:

$$
\begin{array}{r}
\Sigma \mathbf{u}(\mathbf{y})=\mathbf{F}^{u}\left(\mathbf{y}, \Sigma \mathbf{t}, \Delta \mathbf{u}, \mathbf{P}^{0}, \mathbf{P}^{1}, M^{0}, M^{1}\right) \\
\Delta \mathbf{t}(\mathbf{y})=\mathbf{F}^{t}\left(\mathbf{y}, \Sigma \mathbf{t}, \Delta \mathbf{u}, \mathbf{P}^{0}, \mathbf{P}^{1}, M^{0}, M^{1}\right)
\end{array}
$$

Here $\mathbf{P}^{0}, M^{0}, \mathbf{P}^{1}$ and $M^{1}$ are the resultant force and moment applied at the left and right ends of a thin inclusion, respectively. The inclusion model given by Eq. (2) is used as a basic one for description of the edges of the branched inhomogeneities.

According to Pasternak and Sulym (2011), to study a thin branched inclusion the latter is described by the undirected graph $G(V)$, which edges $E_{j i}=E_{i j}=\left(v_{i}, v_{j}\right), v_{i}, v_{j} \in V$ correspond to the non-branched links of the inclusion. It is assumed that the graph $G(V)$ contains no loops $\left(E_{i i}=\left(v_{i}, v_{i}\right) \in 0\right)$ and no isolated vertices. Nevertheless, the absence of loops does not mean that one cannot model the stress state of solids with closed thin inclusions. The later can be described with a closed graph, which contain at least two vertices. Those vertices, which join two or more edges, are further called nodes, and the other are called the free ends of a branched inclusion. Each of the edges $E_{i j}$ can be modeled using various variants of the generalized inclusion model given by Eq. (2).

While studying the doubly periodic problems one should consider two possible cases of interaction of the edges of the branched inclusion: 1) inside the representative volume element, and 2) between the inhomogeneities at the interface of two neighbor representative elements.

To study the first case one can directly use the algorithm developed by Pasternak and Sulym (2011). In particular, if a thin branched inclusion consists of $n$ edges joined with $m \leq n$ nodes and the contact between inclusion's edges is perfect, then at the each of $m$ nodes the following balance equations should be satisfied:

$$
\sum_{k=1}^{q} \mathbf{P}^{k}=\mathbf{0}, \quad \sum_{k=1}^{q} M^{k}=0,
$$

where $q$ is a number of edges of the inclusion, which are joined by the node; $\mathbf{P}^{k}$ and $M^{k}$ are the force and the moment applied at the end of the $k$-th edge included by the vertex. Besides, the continuity conditions for displacements at the node can be satisfied by equating the average values of displacements $\mathbf{u}=\frac{1}{2} \sum \mathbf{u}$ and rotation angles $\omega$ of the edges united by the node:

$\mathbf{u}^{1}=\mathbf{u}^{2}=\ldots=\mathbf{u}^{q}, \omega^{1}=\omega^{2}=\ldots=\omega^{q}$.

The rotation angles (the slope) of the inclusion's lines can be determined, for example, using the Euler-Bernoulli beam equation.

Equations (1), (2) together with relations (3), (4), which produce $3 q$ additional equations for determination of $3 q$ unknown components of contact force ; $\mathbf{P}^{k}$ vectors and moments $M^{k}$ at the nodes of the branched inclusion, can be used for studying doubly periodic problems for inhomogeneous solids, which representative volume elements contain internal thin inclusions.

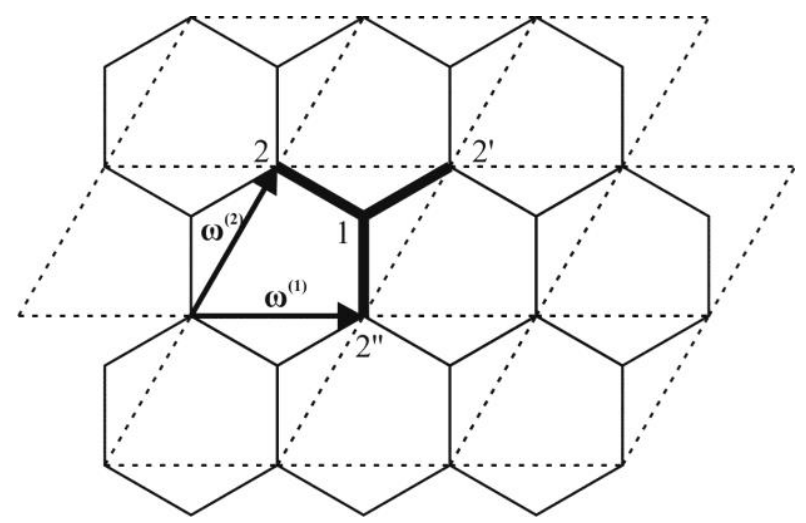

Fig. 2. Doubly periodic set of the thin inclusions joined the interface of neighbor representative volume elements

As for the case of thin branched inclusions, which are joined at the interface of neighbor representative volume elements, 
the displacement continuity conditions (4) are more complicated.

For example, consider honeycomb with hexagonal cells filled with honey (Fig. 2). The walls of the cells can be considered as thin inclusions and the honey represents the elastic medium. The geometry of this doubly periodic problem thus can be set by means of three rectilinear branches of inclusion of the length $a$ each, which repeats with the fundamental periods $\boldsymbol{\omega}^{(1)}=$ $[\sqrt{3 a}, 0]^{\mathrm{T}}, \boldsymbol{\omega}^{(1)}=\frac{1}{2}[\sqrt{3 a}, 3 a]^{\mathrm{T}}$.

For the internal node 1 inside the representative volume element (Fig. 2) relations (3), (4) can be used without any cautions. As for the node 2 formed by the ends $2-2 '-2$ " of the edges of the thin inclusion, the balance equations (3) for forces and moments are also satisfied. However, due to the quasi-periodicity of the displacements, the displacement continuity conditions for the joints at the interface of the neighbor representative elements should account for the cyclic constants. In particular, for the set of inclusions depicted in Fig. 2 the displacement continuity conditions write as:

$\mathbf{u}^{2^{\prime}}=\mathbf{u}^{2}+\delta \mathbf{u}^{(1)}, \mathbf{u}^{2^{\prime}}=\mathbf{u}^{2^{\prime \prime}}+\delta \mathbf{u}^{(2)}$

$\omega^{2}=\omega^{2^{\prime}}, \omega^{2}=\omega^{2 "}$

Here $\delta \mathbf{u}^{(1)}$ and $\delta \mathbf{u}^{(2)}$ are the cyclic constants of the displacement vector along the corresponding fundamental periods. In case of a perfect contact between inclusions and the medium these constants can be determined through the external problem. According to Pasternak (2012), they are defined with the following integral identities:

$$
\begin{aligned}
& \delta \mathbf{u}^{(k)}=\sum_{j} \frac{\partial}{\partial x_{j}} \mathbf{I}^{\infty}(\mathbf{x}) \omega_{x_{j}}^{(k)}+\delta \hat{\mathbf{u}}^{(k)}, \\
& \delta \hat{\mathbf{u}}^{(1)}=0, \\
& \delta \hat{\mathbf{u}}^{(2)}=\int_{\Gamma_{0}^{+}}\left[\mathbf{U}^{*}(\mathbf{x}) \Sigma \mathbf{t}^{0}(\mathbf{x})-\mathbf{T}^{*}(\mathbf{x}) \Delta \mathbf{u}^{0}(\mathbf{x})\right] d \Gamma(\mathbf{x}) .
\end{aligned}
$$

The kernels $\mathbf{U}^{*}(\mathbf{x})$ and $\mathbf{T}^{*}(\mathbf{x})$ are regular.

The obtained system of the boundary integral equations (1)-(6) is suitable for the solution by the modified boundary element method (BEM) of Pasternak (2011). Besides, obtained equations allow determination of the effective mechanical properties of composite materials with the regular structure based on the BEM using the approach of Pasternak (2012).

The BEM numerical implementation should account for the stress square root singularity at the free ends of a thin inclusion. This singularity can be accounted for by utilizing special shape functions (Pasternak, 2011) for modeling of displacement and stress discontinuities at the ends of the discontinuity line $\Gamma_{C}$, which replaces the inhomogeneity. Moreover, according to Pasternak (2011), the strength of the fields' singularity at tips of a thin inhomogeneity is described by generalized stress intensity factors (SIF), which are determined through the discontinuity functions in the local rectangular coordinate system with the origin at the tip of an inhomogeneity by the following formulae:

$$
\mathbf{k}^{(1)}=\lim _{s \rightarrow 0} \sqrt{\frac{\pi}{8 s}} \mathbf{L} \cdot \Delta \mathbf{u}(s), \quad \mathbf{k}^{(2)}=-\lim _{s \rightarrow 0} \sqrt{\frac{\pi s}{2}} \mathbf{S}^{\mathrm{T}} \cdot \Sigma \mathbf{t}(s),
$$

where: $\quad \mathbf{k}^{(i)}=\left[K_{2 i}, K_{1 i}\right]^{T}, \quad \Delta \mathbf{u}=\left[\Delta u_{1}, \Delta u_{2}\right]^{T}, \quad \sum \mathbf{t}=$ $\left[\sum t_{1}, \sum t_{2}\right]^{T}, K_{i j}$ the generalized SIF. For a crack $K_{11}=K_{I}$, $K_{21}=K_{I I}, K_{12}=K_{22}=0$, where $K_{I}, K_{I I}$, are classical SIF of the cracks theory; $\mathbf{L}$ and $\mathbf{S}$ are real Barnett-Lothe tensors
(Ting, 1996), which depend only on the material properties of the medium in the local coordinate system.

The displacement vector and the stress function in the local coordinate system $O x_{1}^{\prime} x_{2}^{\prime}$ with the origin $O$ at the inclusion tip and $O x_{1}^{\prime}$ axis directed along a median line are related to the generalized SIF by the following asymptotic formulae (Pasternak, 2011)

$$
\begin{aligned}
& \mathbf{u}=\sqrt{\frac{2}{\pi}} \operatorname{Im}\left\{\mathbf{A}\left\langle\sqrt{Z_{*}}\right\rangle\left(\sqrt{-1} \mathbf{B}^{-1} \mathbf{k}^{(1)}-2 \mathbf{A}^{\mathrm{T}}\left(\mathbf{S}^{\mathrm{T}}\right)^{-1} \mathbf{k}^{(2)}\right)\right\}, \\
& \boldsymbol{\varphi}=\sqrt{\frac{2}{\pi}} \operatorname{Im}\left\{\mathbf{B}\left\langle\sqrt{Z_{*}}\right\rangle\left(\sqrt{-1} \mathbf{B}^{-1} \mathbf{k}^{(1)}-2 \mathbf{A}^{\mathrm{T}}\left(\mathbf{S}^{\mathrm{T}}\right)^{-1} \mathbf{k}^{(2)}\right)\right\} .
\end{aligned}
$$

Here $\mathbf{A}$ and $\mathbf{B}$ are the Stroh matrices (Ting, 1996); $\left\langle\sqrt{Z_{*}}\right\rangle=$ $\operatorname{diag}\left[\sqrt{x_{1}^{\prime}+p_{1} x_{2}^{\prime}} ; \sqrt{x_{1}^{\prime}+p_{2} x_{2}^{\prime}}\right] ; p_{1}$ and $p_{2}$ are the eigenvalues of the Stroh eigenvalue problem (Ting, 1996); $\boldsymbol{\varphi}$ is the stress function vector, which defines the components of the stress tensor at the arbitrary point by the following relations:

$$
\begin{aligned}
& \boldsymbol{\sigma}_{1}=\left[\sigma_{i 1}\right]=-\boldsymbol{\varphi}_{, 2} \\
& =\frac{-1}{\sqrt{2 \pi}} \operatorname{Im}\left\{\mathbf{B}\left\langle p_{*} \sqrt{Z_{*}}\right\rangle\left(\sqrt{-1} \mathbf{B}^{-1} \mathbf{k}^{(1)}-2 \mathbf{A}^{\mathrm{T}}\left(\mathbf{S}^{\mathrm{T}}\right)^{-1} \mathbf{k}^{(2)}\right)\right\}, \\
& \boldsymbol{\sigma}_{2}=\left[\sigma_{i 2}\right]=\boldsymbol{\varphi}_{, 1} \\
& =\frac{1}{\sqrt{2 \pi}} \operatorname{Im}\left\{\mathbf{B}\langle\sqrt{Z} *\rangle\left(\sqrt{-1} \mathbf{B}^{-1} \mathbf{k}^{(1)}-2 \mathbf{A}^{\mathrm{T}}\left(\mathbf{S}^{\mathrm{T}}\right)^{-1} \mathbf{k}^{(2)}\right)\right\} .
\end{aligned}
$$

According to Sulym (2007), the order of stress singularity at the nodes of the branched inclusion is assumed to be weaker than a square root one (the same as and in the theory of branched cracks). Therefore, to model the ends of the edges joined in the node one can use quadratic discontinuous boundary elements. The same concerns nodes at the interface of the neighbor representative volume elements.

\section{NUMERICAL ANALYSIS OF REGULAR SETS OF THIN BRANCHED INCLUSIONS}

Consider plane strain of the isotropic elastic medium (the Poisson ratio equals 0.3 ) containing a doubly periodic set of thin branched inclusions. The inhomogeneities form the rectangular lattice with the fundamental periods $\boldsymbol{\omega}^{(1)}=[d, 0]^{\mathrm{T}}$ and $\boldsymbol{\omega}^{(2)}=[0,2 d]^{\mathrm{T}}$. Inclusions have the shape of the l-beam cross-section with the width of $2 a$ and the height of $4 a$ (Fig. 3). The thickness of inclusions' edges equals $2 h(h=0.01 a)$. The medium is loaded at the infinity with the uniform bi-axial stress $p$.

Fig. 4 depicts the plots of the normalized generalized SIF $K_{i j}^{*}=\frac{K_{i j}}{p \sqrt{\pi a}}$ at the tips of the branched I-beam inclusion and the components $P_{i}^{*}=\frac{P_{i}}{p a}$ of the force vector, and the bending moment $M^{*}=\frac{M}{(p a)^{2}}$ at the point $E$ of the edge $A E$ of the inclusion depending on the relative rigidity $K=\frac{G^{i}}{G}$ of the latter ( $G$ is the shear modulus). The density of the filament of the medium is defined by the parameter $\lambda=\frac{2 a}{d}$. Continuous curves correspond to the density of $\lambda=0.1$, dashed ones $-\lambda=0.5$, 
and dash-dot $-\lambda=0.8$. For $\lambda=0.1$ the difference between the values of SIF from those obtained for the aperiodic problem for a single I-beam inclusion (Pasternak and Sulym, 2011) does not exceed $0.5 \%$ that justifies the reliability of the developed algorithm and verifies the obtained results.

One can see in Fig. 4 that for soft $(k<1)$ inclusions their approaching essentially increases mode I SIF $K_{11}$ (the normal opening mode). Instead of that, mode II SIF $K_{21}$ does not show such obvious dependence: with the increase in the parameter $\lambda$ SIF $K_{21}$ first decreases, and then starts to increase.

For rigid $(k>1)$ elastic inclusions the generalized mode I SIF $K_{12}$ increases with the growth of $\lambda$. Approaching of the inclusions also causes the growth of the longitudinal force $P_{1}$ in the flanges of the I-beam. Instead of that, limiting values of the mode II generalized SIF $K_{22}$ decrease. Besides, at approaching of the inclusions the bending moment $M$ and the shear force $P_{2}$ at the node $E$ of the flange $A E$ also decrease. These values are determinative for the assessment of the strength of the thin inhomogeneity. Thus, approaching of inclusions allows to reduce stresses both outside and inside the inclusion.

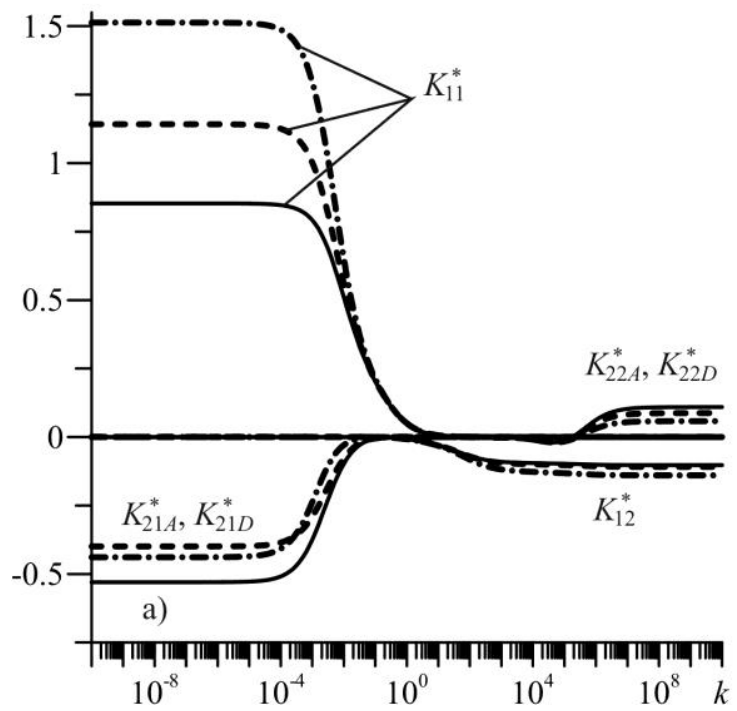

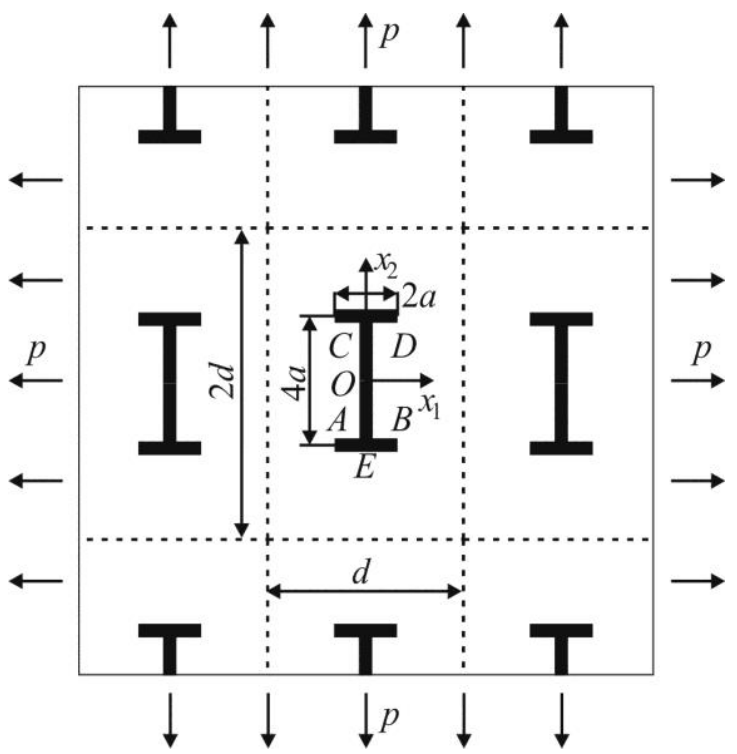

Fig. 3. The elastic medium reinforced with a doubly periodic set of I-beams

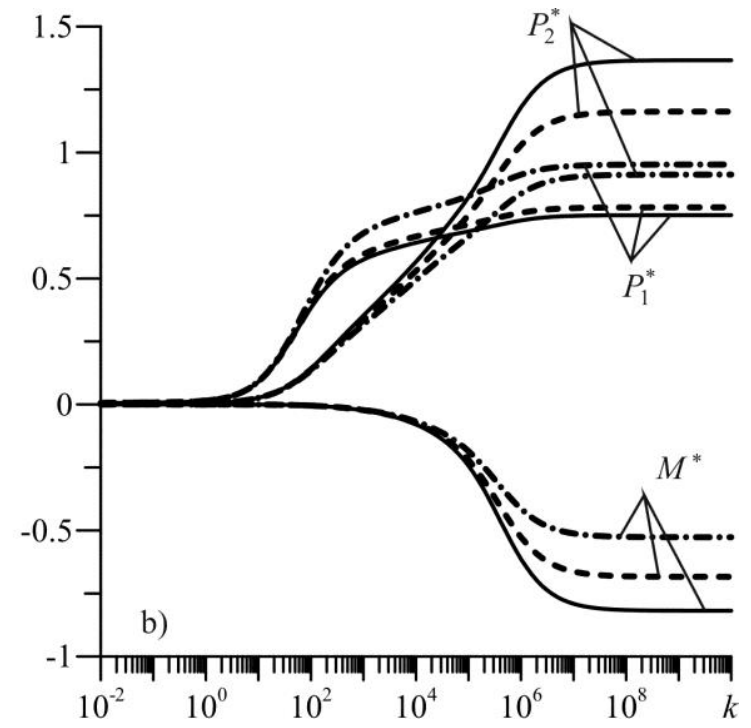

Fig. 4. Stress intensity at the tips of inclusion and the force and bending moment inside it
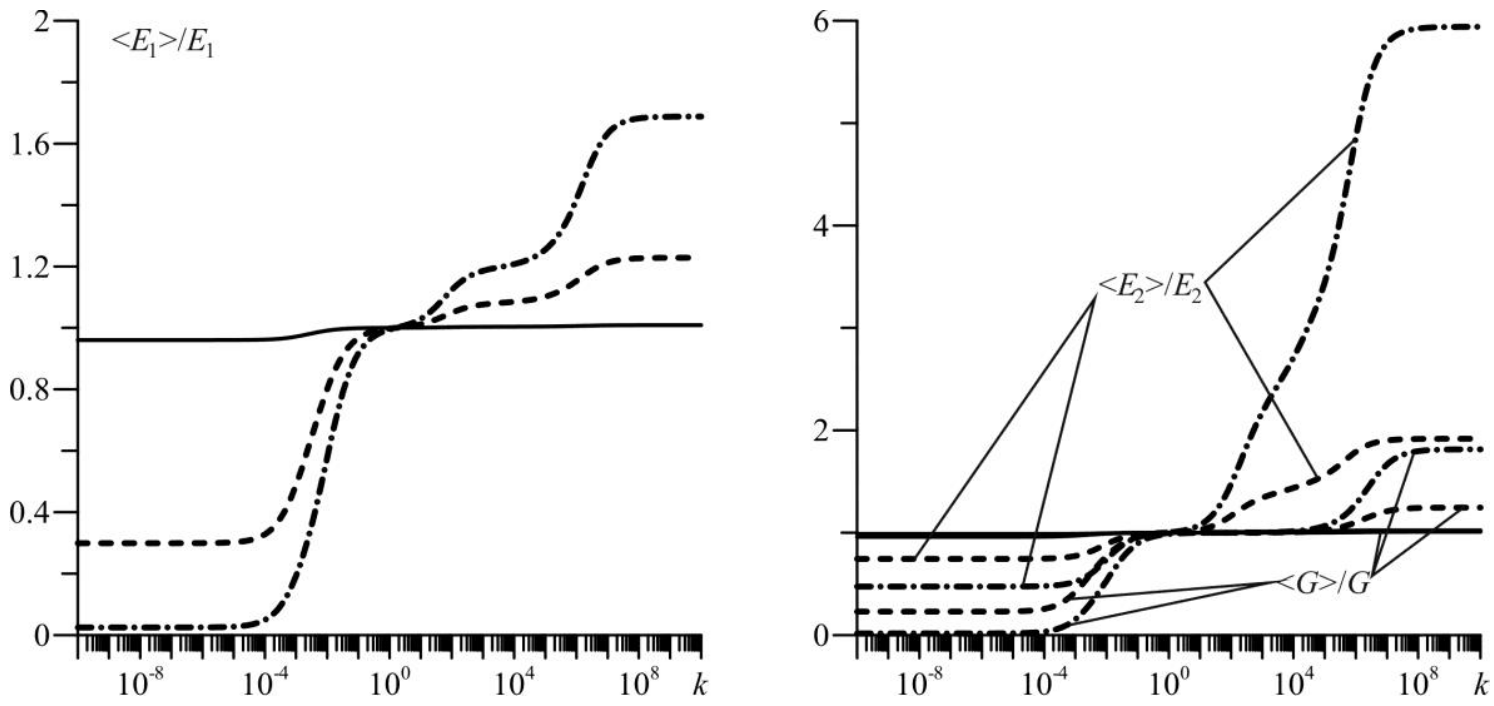

Fig. 5. Effective moduli of the medium reinforced with the I-beams 
Besides stress intensity, developed numeric-analytic approach allows determining of the effective mechanical properties of the medium reinforced by the doubly periodic set of I-beams. For example, Fig. 5 depicts the effective moduli $\left\langle E_{1}\right\rangle$ and $\left\langle E_{2}\right\rangle$, and the effective shear modulus $\langle G\rangle$ for three selected values of the filament density $\lambda$ depending on the relative rigidity $k$ of inclusions.

One can see in Fig. 5 that the reinforced medium is essentially anisotropic. To increase the effective moduli one should reinforce the medium with essentially rigid fibers. It should be noted that the effective moduli $\left\langle E_{1}\right\rangle$ and $\langle G\rangle$ increase with the growth of $k$ in two stages, which is well observed in Fig. 5. Such step-like character of the plot can be related with the influence of bending of the flanges of I-beam. Comparing Fig. 4 and Fig. 5 one can see that the second stage of growth of elastic moduli is related with the increase in the bending moment at the flange.

The most essential increase in the effective modulus is observed for a composite along the vertical axis of the rigid reinforcement I-beam. Moreover, for $\lambda=0.8$ it is possible to increase this modulus up to six times comparing to the unreinforced medium. Taking into account that according to Fig. 4 the generalized SIF $K_{22}$, shearing force and the bending moment at the flange decrease, such reinforcing can be considered as a favorable one.

\section{CONCLUSIONS}

The proposed approach for studying of doubly periodic sets of thin branched inclusions allows not only to determine stress concentration and intensity in composites with regular reinforcement, but also to assess the effective mechanical properties of such materials. Thus, the developed technique can be used as a tool for designing and optimization of the composite materials reinforced with thin filament, in particular, with a standard shapes. The considered example for a medium reinforced with the I-beams justified the efficiency of the proposed approach and allowed determining effective moduli of such composite. In particular, it is observed that approaching of such thin rigid reinforcements allows both to increase effective moduli and to decrease internal stress concentration and intensity.

\section{REFERENCES}

1. Antipov Yu.A., Popov G.Ya., Yatsko S.I. (1987), Solution of the problem of stress concentration around intersecting defects by using the riemann problem with an infinite index, Journal of Applied Mathematics and Mechanics, 51(3), 357-365.

2. Dolgikh V.N., Fil'shtinskii L.A. (1979), Model of an anisotropic medium reinforced by thin tapes, Soviet Applied Mechanics, 15(4) 292-296.

3. Grigoryan E.H., Torosyan D.R., Shaghinyan S.S. (2002), A problem for an elastic plane containing a cross-like inclusion, Mechanics. Proceedings of National Academy of Sciences of Armenia, 55 (1), 6-16.

4. Kosmodamianskij A.S. (1976), Naprâžennoe sostoânie anizotropnyh sred s otverstiâmi ili polostâmi, Vyšča škola, Kyiv.

5. Osiv O.P, Sulym H.T. (2002), Antyploska deformaciâ seredovyšča zi zlučenymy pružnymy vklûčennâmy, Mehanika i fizyka rujnuvannâ budivelnuh materialiv $i$ konstrukcij, 5, 154-164.

6. Osiv 0., Sulym G. (2001), Antiplane deformation of isotropic medium with connected elastic ribbon-like inclusions, Abstracts of the Fourth Polish-Ukrainian Conference "Current Problems in Mechanics of Nonhomogeous Media" (Łódż, 4-8 Sept., 2001), Technol. Univ. of Łódż, Łódż.

7. Pasternak la. (2011), Coupled 2D electric and mechanical fields in piezoelectric solids containing cracks and thin inhomogeneities, Engineering Analysis with Boundary Elements, 35(4), 678-690.

8. Pasternak la. (2012), Doubly periodic arrays of cracks and thin inhomogeneities in an infinite magnetoelectroelastic medium, Engineering Analysis with Boundary Elements, 36(5), 799-811.

9. Pasternak la., Sulym H. (2011), Ploska zadača teorij pružnosti anizotropnogo tila z tonkymy gillâstymy pružnymy vklûčennâmy, Visnyk Ternopilskogo NTU, 16(4) 23-31.

10. Pasternak la., Sulym H. (2013), Stroh formalism based boundary integral equations for 2D magnetoelectroelasticity, Engineering Analysis with Boundary Elements, 37(1), 167-175.

11. Popov V.G. (1993), Dynamic problem of the theory of elasticity for a plane containing a rigid cruciform inclusion, Journal of Applied Mathematics and Mechanics, 57(1), 125-131.

12. Šackyj I.P, Kundrat A.M. (2004), Antyploska deformaciâ pružnogo prostoru zi zvâzanymy žorstkymy stričkovymy vklûčennâmy, Dopovivdi NAN Ukrajiny, 11, 55-60.

13. Sulym H.T. (2007), Osnovy matematyčnoj teorij termopružnoj rivnovagy deformivnyh til z tonkymy vklûčennâmy, Dosl.-vydav. centr NTŠ, L'viv.

14. Ting T.C.T. (1996), Anisotropic elasticity: theory and applications, Oxford University Press, New York. 